\title{
An Evolutionary Strategy with Machine Learning for Learning to Rank in Information Retrieval
}

\author{
O. Ibrahim • D. Landa-Silva
}

Received: date / Accepted: date

\begin{abstract}
Learning to Rank (LTR) is one of the problems in Information Retrieval (IR) that nowadays attracts attention from researchers. The LTR problem refers to ranking the retrieved documents for users in search engines, question answering and product recommendation systems. There is a number of LTR approaches based on machine learning and computational intelligence techniques. Most existing LTR methods have limitations, like being too slow or not being very effective or requiring large computer memory to operate. This paper proposes a LTR method that combines a $(1+1)$-Evolutionary Strategy with machine learning. Three variants of the method are investigated: ES-Rank, IESR-Rank and IESVMRank. They differ on the mechanism to initialize the chromosome for the evolutionary process. ES-Rank simply sets all genes in the
\end{abstract}

Osman Ali Sadek Ibrahim

School of Computer Science

ASAP Research Group

The University of Nottingham, UK

CS. Dept., Minia University, Egypt

E-mail: osmaneg200@gmail.com

Dario Landa-Silva

School of Computer Science

ASAP Research Group

The University of Nottingham, UK

E-mail: dario.landasilva@nottingham.ac.uk initial chromosome to the same value. IESRRank uses linear regression and IESVM-Rank uses support vector machine for the initialization process. Experimental results from comparing the proposed method to fourteen other approaches from the literature show that IESRRank achieves the overall best performance. Ten problem instances are used here, obtained from four datasets: MSLR-WEB10K, LETOR 3 and LETOR 4. Performance is measured at the top-10 query-document pairs retrieved, using five metrics: Mean Average Precision (MAP), Root Mean Square Error (RMSE), Precision (P@10), Reciprocal Rank (RR@10) and Normalized Discounted Cumulative Gain (NDCG@10). The contribution of this paper is an effective and efficient LTR method combining a listwise evolutionary technique with point-wise and pair-wise machine learning techniques.

Keywords Learning to Rank · Evolution Strategy · Linear Regression · Support Vector Machine

\section{Introduction}

Ranking the retrieved documents responding to the user query, with respect to the relevance of the documents for the query, is an important task in Information Retrieval (IR). In the 
early IR research, unsupervised scoring methods such as TF-IDF, Okapi-BM25 and language models among others were used (Manning et al., 2008). Using only one scoring method in IR systems is not very efficient. Moreover, the accuracy of results produced by learning models such as Okapi-BM25 and language models is dependent on the relevance judgment (Tonon et al., 2015; Urbano, 2016; Ibrahim and Landa-Silva, 2016). This inspires the need for using more than one scoring method for ranking retrieved documents with respect to the user queries. In addition, it is also important that other features such as the business importance of the documents on the web and the host server among other desirable features are considered for the ranking of documents. Recently, Qin et. al. proposed a new trend in the research on ranking documents by producing the LETOR datasets (Qin et al., 2010). These datasets are distilled benchmarks from search engines and from the well-known TREC conference collections. These benchmarks contain more than one scoring weighting scheme as part of the benchmark features. They also contain some other features that indicate the importance of the documents on the web. The documents in these datasets were mapped into fully judged query-document pairs for Learning to Rank (LTR) research problems.

Previous work described an LTR approach called ES-Rank based on an evolutionary strategy (Ibrahim and Landa-Silva, 2017). The performance of that method was competitive when compared to fourteen other approaches from the literature. ES-Rank produced better results but only in about $30 \%$ of the problem settings. Further research has resulted in the incorporation of machine learning techniques into ES-Rank as described in this paper. Moreover, a more thorough experimental comparison is conducted here to evaluate the performance of the proposed method using a larger set of problem instances and five performance metrics.

The intended contribution of this paper is to present an effective and efficient method for
LTR that combines an evolutionary strategy with machine learning techniques. The proposed method is an evolutionary strategy that evolves a vector of weights where each weight represents a desirable document feature. Three methods to initialize the vector of weights (chromosome) are investigated here: simply setting all weights to zero, initializing with Linear Regression and initializing with a Support Vector Machine. In order to assess the performance of the proposed method at top-10 query-document pairs retrieved, the following five metrics are used in a comparative analysis to fourteen stateof-the-art LTR methods from the literature: Mean Average Precision (MAP), Root Mean Square Error (RMSE), Normalized Discounted Cumulative Gain (NDCG), Reciprocal Ranking (RR) and Precision (P) (Liu, 2011; Li, 2014). Experimental results in this paper show that the proposed method performs very well and that the better initialization technique is Linear Regression (LR) as it helps to achieve the best overall results. Furthermore, most of the other methods compared consume very long computation time (up to 7 hours) while the proposed method is much faster. Another important feature of the proposed method is that the computer memory required for it to operate is modest, only in the order of $(2 \times M)$ where $M$ is the number of features in the training dataset.

The rest of the paper is organized as follows. Section 2 provides a discussion on the background of the LTR problem in IR, while section 3 presents a brief literature review of related work in this subject. The proposed LTR method is described in section 4. Experimental results are presented in section 5, while conclusions and proposed future work are given in Section 6.

\section{Background}

In the context of Information Retrieval (IR), a LTR dataset consists of query-document pairs for a large number of queries (Qin et al., 
Table 1: Query-Document Pairs Representation in Learning to Rank (LTR)

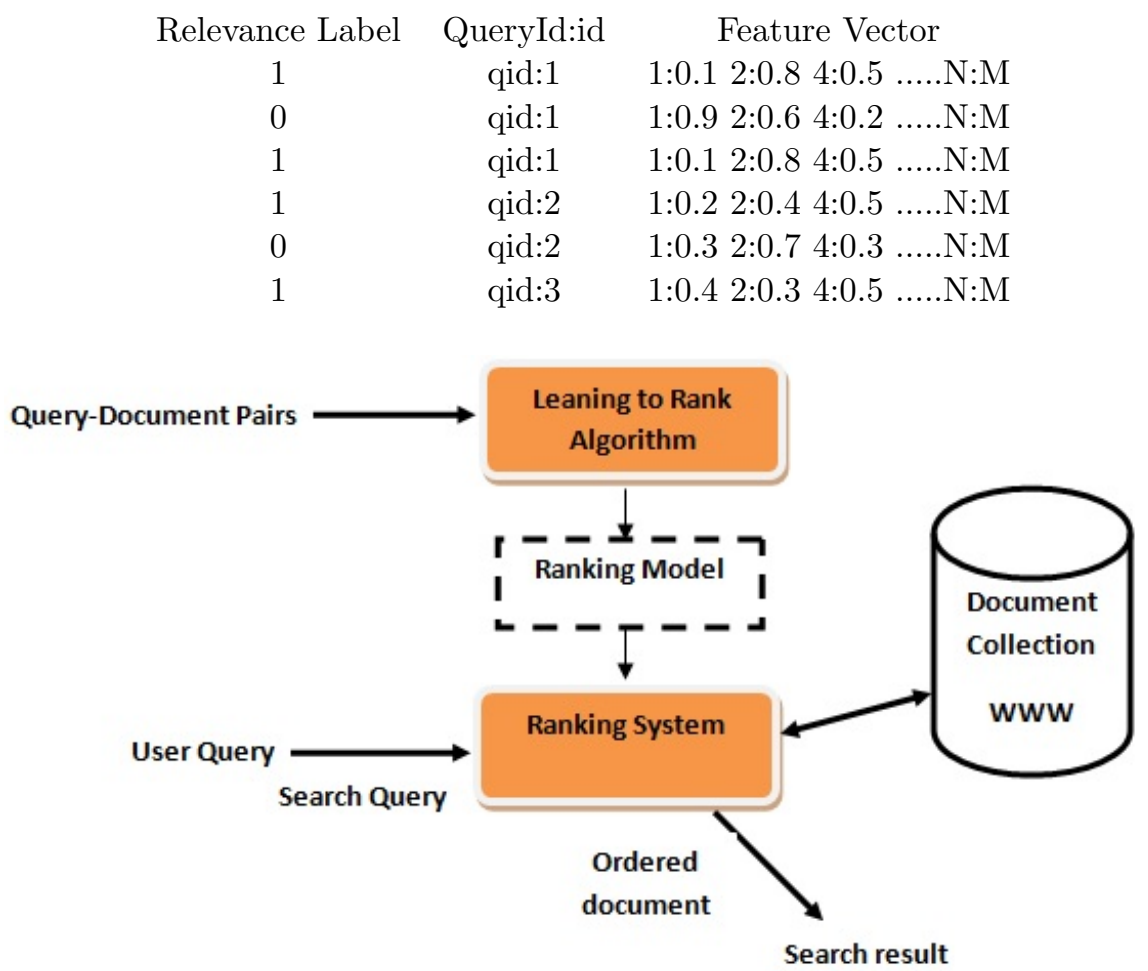

Fig. 1: Architecture of a Learning to Rank Approach as Discussed in (Liu, 2009).

2010). Table 1 shows a representation of several query-document pairs. Each pair contains a relevance label indicating the relevance degree of the document for each query. In most cases, the relevance labels are binary where 1 means relevant and 0 means irrelevant. There is also a query identifier (id) indicating the corresponding query for each query-document pair. The feature vector refers to $M$ other features such as Term-Weighting Scores (e.g. TFIDF, Okapi-BM25 and Language Models (Qin et al., 2010)), PageRank and Host Server Importance, features associated to each querydocument pair in the LTR dataset. Each feature in the Feature Vector has the form FeatureID:FeatureValue, where FeatureValue is the contribution value of this feature in the query-document pair. The dataset itself is divided into $N$ folds (usually LTR datasets have
5 folds) and each fold contains training, validation and testing sets for the query-document pairs. These folds are useful for examining the behaviour and predictive performance of LTR methods by applying them on test sets different to the training sets. More details about the organisation of LTR datasets and querydocument pairs are given in (Qin et al., 2010; Qin and Liu, 2013).

Recently, LTR as a method based on supervised learning, has been widely used in IR to produce ranking functions based on the training datasets. The ranking function is used to rank the retrieved documents in response to the user query. Figure 1 shows the general LTR approach architecture that most learning-based approaches follow to deal with the IR ranking problem. It starts with the training set made of query-document pairs be- 
ing the input to a computational intelligence or machine learning technique (Li, 2014). The ranking model or ranking function is created and then used to rank the search results for the user queries. The ranking model can also be used in the test phase to measure the predictive performance of the ranking algorithm on the test datasets. The resulting ranking system will produce an ordered list of documents retrieved from the document collection in response to the search. The next section reviews some of the existing LTR approaches in order to set the context for the method proposed in this paper.

\section{Related Work}

There are three categories of LTR methods (Liu, 2009): (1) the point-wise method, (2) the pair-wise method and (3) the list-wise method. These categories are based on the loss function or fitness function measurements. The point-wise approach views each single object (query-document pair) as the learning instance. Examples of point-wise approach are Linear Regression (Yan and Su, 2009), Boosting (Freund et al., 2003), Gradient Boosted Regression Trees (MART or GBRT) (Friedman, 2001; Mohan et al., 2011) and Random Forest (RF) (Breiman, 2001). The pair-wise approach views the pair of objects (two query-document pairs for the same query) as the learning instance. Examples of the pair-wise approach are RankNET (Burges et al., 2005) which is based on neural networks, as well as RankBoost and SVMRank (Li, 2014) which are based on support vector machines. The list-wise approach takes the entire list of objects retrieved (the list of query-document pairs for each query) as the learning instance. Examples of the list-wise approach are ListNET (Cao et al., 2007) which is based in neural networks, RankGP (Lin et al., 2012; Mick, 2016), Coordinate Ascent (Metzler and Bruce Croft, 2007), AdaRank (Xu and Li, 2007) and RankGPES
(Islam, 2013).

Although list-wise methods have been shown to perform better regarding accuracy than point-wise and pair-wise approaches (Cao et al., 2007), the need to improve the performance of LTR approaches has motivated researchers to propose hybrid methods as well. For example, Sculley proposed an approach (CoRR) combining linear regression (pointwise) with support vector machine (pair-wise) (Sculley, 2010). That approach is implemented in the Sofia-ml package and while it executes in reasonable computational time, its performance in terms of NDCG and MAP is limited. In order to achieve better NDCG, Mohan et al. proposed a hybrid machine learning approach for initializing GBRT using Random Forest (Mohan et al., 2011). However, experiments showed that their approach consumes too much run-time compared to other approaches from the literature (Dang, 2016; $\mathrm{Li}, 2014$ ). Two other hybrid approaches are LambdaRank and LambdaMART which combine pair-wise with list-wise methods (Burges, 2010). LambdaRank is based on RankNET while LambdaMART is the boosted tree from LambdaRank. Both LambdaMART and LambdaRank have shown better performance regarding IR accuracy than the method by Mohan et. al. on the Yahoo! LTR Challenge (Chapelle and Chang, 2011). Most of the LTR approaches still have some limitation on the computational run-time or the achieved accuracy of the predictive results. However, the combination of list-wise and point-wise techniques has shown to be promising. Muahmmed and Carman conducted experiments combining list-wise with point-wise Random Forest (Hybrid RF) showing that the their hybrid outperformed other methods both in computational run-time and accuracy.

In a previous paper, the ES-Rank method was proposed to tackle the LTR problem in IR (Ibrahim and Landa-Silva, 2017). The method uses a $(1+1)$ Evolutionary Strategy to evolve a single vector (the ranking 
function) over a number of generations. A preliminary investigation of ES-Rank showed that the method performed well against other fourteen methods from the literature on three datasets and with respect to mean average precision (MAP), normalized discounted cummulative gain (NDCG) and computational run-time. For example, the computational run-time of IGBRT (rt-rank package) or Coordinate Ascent (Ranklib package) on the MSLR-WEB10K fold was over 9 hours while ES-Rank used just around 30 minutes. However, linear regression was even faster on that dataset using less than 3 minutes. Most of the other methods consumed more than one hour of computation run-time on the MSLR-WEB10K fold. Observing that linear regression, a point-wise technique, was very fast while still achieving reasonable accuracy, motivated the research presented in this paper. The previous paper used two fitness evaluation metrics and three test datasets.

This paper presents improved versions of ES-Rank, called IESR-Rank and IESVMRank, that incorporate machine learning techniques. Hence, the hybrid LTR methods proposed here combine the list-wise approach of ES-Rank with the point-wise approach of linear regression and the pair-wise approach of support vector machine for even better accuracy and lower computational run-time. This paper also includes a more extensive evaluation of ES-Rank using five evaluation metrics and ten LTR datasets.

\section{The Proposed LTR Approach}

The proposed LTR methodology uses a $(1+1)$ Evolutionary Strategy (ES) for evolving the ranking function, due to the proven capability of evolutionary strategies to effectively and efficiently converge towards a better solution (Beyer and Schwefel, 2002). A (1+1)-ES works on a population of size two, the current solution (parent) and the candidate solution (offspring) which results from mutating the parent. Only if the offspring is at least as good as the parent, it replaces the parent for the next generation, otherwise the offspring is disregarded. A solution or chromosome is a vector of weights all together representing the ranking function being evolved. It is well-known that choosing an appropriate initial solution in evolutionary techniques is an important issue (Diaz-Gomez and Hougen, 2007). Three ways to create the initial parent are investigated here. One is to set all weights to the same value of zero, another one uses Linear Regression (LR), the third one uses Support Vector Machine. Experiments later in this paper show that using Linear Regression or Support Vector Machine for parent initialization helps ES-Rank to converge towards better solutions.

Algorithm 1 outlines the ES-Rank method. The input is the training set of querydocument pairs or feature vectors and the output is a linear ranking function. The chromosome ParentCh is a vector of $M$ genes, where each gene is a real number representing the importance of the corresponding feature for ranking the document. Steps 1 to 4 initialize the chromosome vector by setting each gene to a value of 0 . The Boolean variable Good used to indicate whether repeating the mutation process from the previous generation is set to FALSE in Step 5. A copy of ParentCh is made into OffspringCh in step 6. The evolution process for MaxGenerations generations (1300 in this paper) starts in Step 7 and ends in Step 24. Steps 8 to 16 show the strategy to control the mutation process by choosing the number of genes to mutate $(R)$, the actual genes to mutate and the mutation step. The mutation step is determined using Equation (1) where $\operatorname{Gaussian}(0,1)$ is a random Gaussian number with 0 mean and 1 standard deviation, and $\operatorname{Cauchy}(0,1)$ is a cumulative distributed Cauchy random number with value between 0 and 1. 


$$
\begin{array}{r}
\text { Mutated_Gene_i } i=\text { Gene_ } i \\
+\operatorname{Gaussian}(0,1) * \exp (\text { Cauchy }(0,1))
\end{array}
$$

The mutation step defined by Equation 1 was chosen based on preliminary experiments in which several ways of combining the Gaussian and Cauchy numbers were tried. The combinations tried involved adding, subtracting and multiplying these numbers. Both random and probabilistic mutation rates were tried in the preliminary experiments. Among the various combinations tried, the one expressed by Equation 1 provided the best performance for ES-Rank. A mutation process that is successful (produces a better offspring) in generation $(G-1)$ is replicated in generation $G$ as shown in Step 9. Otherwise the parameters of the mutation process are reset as shown in Steps 11 to 15 . Steps 17 to 23 select between the ParentCh and the OffspringCh according to their fitness function values. Finally, ES-Rank returns the ranking function in Step 25, defined by the transpose of the evolved vector of feature weights and the query-document pairs. The computational complexity of this algorithm is $\Omega(N * n * \log (R))$, where $N$ is the number of training query-document pairs, $n$ is the number of evolving iterations and $R$ is the number of genes in the chromosome. The link for ES-Rank library package is: IESRank.zip.

Instead of the simple initialization process in steps 1 to 4 of Algorithm 1, Linear Regression (LR) and Support Vector Machine (SVM-Rank) are used now. That is, the genes in the ParentCh vector take the weight values that result from the least square LR or SVM-Rank models (Dang, 2016; Joachims, 2016a). Incorporating these machine learning techniques into an evolutionary approach is a novel idea within the LTR domain. The reason for choosing LR and SVMRank is as well as ES-Rank, they produce linear ranking models, while other techniques produce non-linear ranking models or they have high computational run-time.

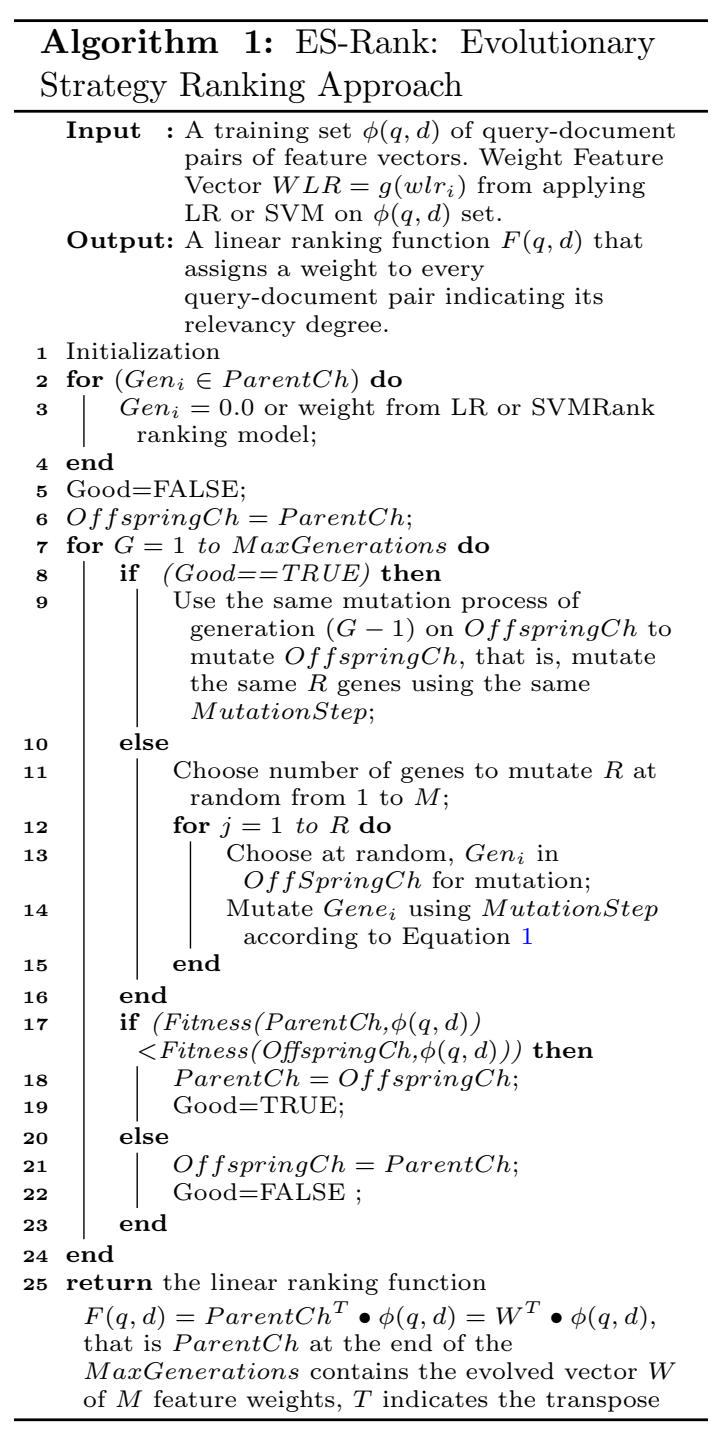

The run-time efficiency of the proposed method also allows for all training instances to be used in each learning iteration. Most other LTR techniques do not do that and instead they use sampling methods for learning and checking the quality of the proposed ranking models. However, sampling methods such as bootstrap Bagging or Boosting cause overfitting and under-fitting problems. The proposed method evolves better ranking models with smooth fitting and better performance regarding run-time and accuracy. 
In Bagging (Bootstrap Aggregation) methods such as RF, the training dataset is divided into a number of bags. Then, a training ranking model is produced for each training sample the average ranking model from all learning ranking models is taken as the general ranking model of the RF technique. This method is used to reduce the variance of the learning ranking model on the training dataset and hence reduce the over-fitting on predictive test dataset. However, this averaging method limits the increase in performance of the ranking model. On the other hand, random sampling takes sample data from the training dataset for learning the ranking model in each learning iteration of the machine learning technique and this may cause over-fitting by high variance model representation or under-fitting by high bias as mentioned in (Brownlee, 2017). This issue also affects the performance of the ranking model. In the following subsections, SVMRank (LTR with support vector machines) and LR (LTR with linear regression) are described in more detail.

\subsection{SVMRank: Support Vector Machine for} LTR

Joachims proposed a pairwise approach called SVMRank for LTR based on a Support Vector Machine (Joachims, 2016a). The approach compares every two query-document pairs in order to rank them in a retrieved querydocument pair list. This approach uses the error rate between the actual ranking and the ranking from its model as a loss function. The objective of the SVMRank technique is to minimise the loss function value between the actual relevance labels and the ranking model labels on the training dataset. This approach produces a linear ranking model of weights. Assume the vector of weights that are adjusted by the SVMRank technique is $\vec{w}$. The ranking model is represented by $f_{\vec{w}}(q)$, where $q$ is the query set of the training data. The ranking of two documents $d_{i}$ and $d_{j}$ that have querydocument pairs $\Phi\left(q, d_{i}\right)$ and $\Phi\left(q, d_{j}\right)$ can be represented by:

$\left(d_{i}, d_{j}\right) \epsilon f_{\vec{w}}(q) \Leftrightarrow \vec{w} \Phi\left(q, d_{i}\right)>\vec{w} \Phi\left(q, d_{j}\right)$

If the training set contains $n$ queries, the target of the SVMRank is to find the weight vector $\vec{w}$ that maximises the number of fulfilled inequalities in:

$$
\begin{array}{r}
\left(d_{i}, d_{j}\right) \epsilon r_{1}^{*}: \vec{w} \Phi\left(q_{1}, d_{i}\right)>\vec{w} \Phi\left(q_{1}, d_{j}\right) \\
\ldots \ldots \\
\ldots . \\
\left(d_{i}, d_{j}\right) \epsilon r_{n}^{*}: \vec{w} \Phi\left(q_{n}, d_{i}\right)>\vec{w} \Phi\left(q_{n}, d_{j}\right)
\end{array}
$$

This direct generalisation in Equation (3) for Equation (2) shows that this problem is a complex (NP-hard) problem to solve. However, it can be simplified based on the classification problem using SVM. Thus, the optimisation problem of SVMRank can be represented as follows:

minimise: $\quad V(\vec{w}, \vec{\xi})=\frac{1}{2} \vec{w} \cdot \vec{w}+C \sum \xi_{i, j, k}$

subject to:

$$
\begin{aligned}
&\left(d_{i}, d_{j}\right) \epsilon r_{1}^{*}: \vec{w} \Phi\left(q_{1}, d_{i}\right) \geq \vec{w} \Phi\left(q_{1}, d_{j}\right)+1-\xi_{i, j, 1} \\
& \ldots \ldots \\
& \ldots . . \\
&\left(d_{i}, d_{j}\right) \epsilon r_{n}^{*}: \vec{w} \Phi\left(q_{n}, d_{i}\right) \geq \vec{w} \Phi\left(q_{n}, d_{j}\right)+1-\xi_{i, j, n} \\
& \forall i, \forall j \text { and } \forall k: \xi_{i, j, k} \geq(\overleftarrow{\Phi})
\end{aligned}
$$

where $C$ is a constant that adjusts the margin size against the training error and $\xi_{i, j, k}$ is the slack variable. Thus, the problem is to minimise the upper bound of $\sum \xi_{i, j, k}$. This problem is a convex problem that has no local optima. For clarification, constraints in Equation (5) can be re-arranged as:

$$
\vec{w}\left(\Phi\left(q_{k}, d_{i}\right)-\Phi\left(q_{k}, d_{j}\right)\right) \geq 1-\xi_{i, j, k},
$$

Initially, Joachims proposed a support vector machine called svmlight library package 
(Joachims, 2016b). However, this package was slower than other LTR techniques. Thus, he later proposed a new library package for ranking called SVMRank (Joachims, 2016a). SVMRank is faster because it does not include all query-document pairs of the training set in each learning iteration.

\subsection{Linear Regression for LTR}

The Linear Regression (LR) technique was introduced in the Ranklib library package (Dang, 2016), but there is no paper discussing its comparison to other LTR techniques. The method used in Ranklib is the least square LR technique (Miller, 2006). In this method, the ranking model weight vector is chosen based on minimising the total distance between the ground truth labels of the training query-document pairs and the labels produced by ranking the ranking model. The objective of the ranking model produced by the LR technique is to minimise loss $=\frac{1}{N} \sum_{j=1}^{N} \mid y_{j}-$ $\sum_{i=1}^{n}\left(w_{i} x_{i j}\right) \mid$. In this equation, $N$ is the number of query-document pairs in the training set, $n$ is the number of features in each querydocument pairs, $w_{i}$ is the weight for feature $i$ in the ranking model proposed by LR and $x_{i j}$ is the feature value for feature $i$ in querydocument pair $j$. Finally, $y_{j}$ is the ground truth label for query-document pair $j$. From our experiments, the LR technique in Ranklib is the fastest approach, but it is not the most efficient one within the Ranklib package.

\section{Experimental Study and Evaluation}

This section presents a comprehensive experimental study comparing the performance of the proposed LTR approach to fourteen other methods both in terms of accuracy and computational run-time. Accuracy is measured using five metrics described in subsection 5.2: Mean Average Precision (MAP), Normalized Discounted Cumulative Gain (NDCG), Precision (P), Reciprocal Rank (RR) and Root
Mean Square Error (RMSE). In order to assess the performance of a method for LTR, benchmark datasets containing training, validation and test sets are identified. The LTR approach is first applied to the training set in order to learn a ranking function. Then, the performance of the learned ranking function is assessed using the test set to measure the predictive performance of the LTR algorithm.

\subsection{Benchmark Datasets}

The benchmark datasets used in the experiments of this paper are MSLR-WEB10K, LETOR 4 (MQ2007 and MQ2008) and LETOR 3 ( Ohsumed, TD2003, TD2004, HP2003, HP2004, NP2003 and NP2004) (Qin and Liu, 2013; Liu, 2011; Qin et al., 2010). Table 2 outlines the properties of these datasets. The number of query-document pairs and the number of features in the Microsoft Bing Search dataset (MSLR-WEB10K) are much larger than in the LETOR 4 (MQ2007 and MQ2008) or the LETOR 3 (Ohsumed and .Gov) datasets. Each query-document pair contains low-level features such as term frequency and inverse document frequency of the document terms existing in the queries. The low-level features were determined for all document parts (title, anchor, body and whole). There are also high-level features that indicate the similarity matching between the queries and the documents. Furthermore, hybrid features represent the recent research IR models in SIGIR conference papers such as Language Model with Absolute Discounted Smoothing (LMIR.ABS), Language Model with Jelinek-Mercer smoothing (LMIR.JM), Language Model with Bayesian smoothing using Dirichlet priors (LMIR.DIR) and User Click features (Liu, 2011; Qin et al., 2010; Qin and Liu, 2013).

The largest number of queries (10000) is in the MSLR-WEB10K dataset. All the other datasets have less than 1000 queries with the exception of the MQ2007 dataset which 
has 1692. Each query has associated a number of relevant and irrelevant documents, i.e. query-document pairs for each query. The relevance label indicates the relevance degrees for the queries with the documents (querydocument relationship). In most cases, the relevance labels include values of 0 (for irrelevant), 1 (for partially relevant) and 2 (totally relevant). The exception is for the MSLRWEB10K dataset with values (created by the Bing search engine) from 0 (irrelevant) to 4 (perfectly relevant). The LETOR 3 and LETOR 4 datasets were constructed by several research groups working in collaboration (Qin and Liu, 2013; Liu, 2011). To the best of our knowledge, besides the preliminary work reported in (Ibrahim and Landa-Silva, 2017), this paper is the first one to conduct a comprehensive comparison between many LTR approaches considering several accuracy metrics and computational run-time on several very different benchmark datasets.

\subsection{Fitness and Evaluation Metrics}

The following five accuracy metrics are used in this study: MAP, NDCG@10,P@10,RR@10 and RMSE (Baeza-Yates and Ribeiro-Neto, 2011; Li, 2014). Each of them is used as separate fitness function on the training sets and also as the evaluation metric for the ranking functions on the test sets. Each of these metrics is described in detail next.

Let $d_{1}, d_{2}, \ldots, d_{D}$ denote the sorted documents by decreasing order of their similarity measure function value, where $D$ represents the number of retrieved documents. The function $r\left(d_{i}\right)$ gives the relevance value of a document $d_{i}$. It returns 1 if $d_{i}$ is relevant, and 0 otherwise. The Precision per query $q$ for top- $D$ document retrieved $\left(P_{q} @ D\right)$ is defined as follows:

$$
P_{q} @ D=\sum_{i=1}^{D} r\left(d_{i}\right) \cdot \sum_{j=1}^{D} \frac{1}{j}
$$

The Average Precision per query set $Q$ $(A v g P)$ is the average precision values over all queries $Q$. This can be given by the following equation:

$\operatorname{Avg} P=\frac{\sum_{q=1}^{Q} P_{q} @ D}{Q}$

The $A v g P$ value is calculated for a top-D query-document pairs retrieved. The mean of the average precision values for over all querydocument pairs retrieved (MAP) can be given by the following equation:

$M A P=\frac{\sum_{k=1}^{M} \operatorname{Avg} P}{M}$

Where $M$ is number of $A v g P$ points existing in the search result. For considering the graded relevance levels in the datasets for LTR techniques evaluation $r\left(d_{j}\right)$ returns graded relevance value (not binary relevance value as in MAP and $P_{q} @ D$ equations) in Equations 10, 11 and 12 for other fitness evaluation metrics. The Normalized Discounted Cumulative Gain of top-k documents retrieved (NDCG@k) in Equation 10 can be calculated by:

$$
N D C G @ k=\frac{1}{I D C G @ k} \cdot \sum_{i=1}^{k} \frac{2^{r\left(d_{i}\right)}-1}{\log _{2}(i+1)}
$$

where $I D C G @ k$ is the ideal (maximum) discounted cumulative gain of top- $\mathrm{k}$ documents retrieved. The Discounted Cumulative Gain of top-k documents retrieved (DCG@k) can be calculated by the following equation:

$D C G @ k=\sum_{i=1}^{k} \frac{2^{r\left(d_{i}\right)}-1}{\log _{2}(i+1)}$

If all top-k documents retrieved are relevant, the $D C G @ k$ will be equal to $I D C G @ k$.

The Reciprocal Rank at top- $K$ retrieved query-document pairs $(\mathrm{RR} @ \mathrm{~K})$ is as follows:

$R R @ K=\sum_{i=1}^{k} \frac{r\left(d_{i}\right)}{i}$ 
Table 2: Properties of the benchmark datasets used in the experimental study.

\begin{tabular}{|l|l|l|l|l|l|}
\hline Dataset & Queries & Query-Document Pairs & Features & Relevance Labels & No. of Folds \\
\hline MQ2007 & 1692 & 69623 & 46 & $\{0,1,2\}$ & 5 \\
\hline MQ2008 & 784 & 15211 & 46 & $\{0,1,2\}$ & 5 \\
\hline Ohsumed & 106 & 16140 & 45 & $\{0,1,2\}$ & 5 \\
\hline HP2003 & 150 & 147606 & 64 & $\{0,1,2\}$ & 5 \\
\hline TD2003 & 50 & 49058 & 64 & $\{0,1,2\}$ & 5 \\
\hline NP2003 & 150 & 148657 & 64 & $\{0,1,2\}$ & 5 \\
\hline HP2004 & 75 & 74409 & 64 & $\{0,1,2\}$ & 5 \\
\hline TD2004 & 75 & 74146 & 64 & $\{0,1,2\}$ & 5 \\
\hline NP2004 & 75 & 73834 & 64 & $\{0,1,2\}$ & 5 \\
\hline MSLR-WEB10K & 10000 & 1200192 & 136 & $\{0,1,2,3,4\}$ & 5 \\
\hline
\end{tabular}

The Error Rate (Err) is usually used to measure the error of the learning model if it is used on another benchmark different from the training set. It is the subtraction between the training evaluation value to the predictive evaluation value, while the Mean Absolute Error and Root Mean Square Error are calculated by Equations 13 and 14 .

$M A E=\frac{1}{n} \sum_{i=1}^{n}\left|E r r_{i}\right|$

$R M S E=\sqrt{\frac{1}{n} \sum_{i=1}^{n}\left(E r r_{i}\right)^{2}}$

where $n$ is the number of benchmark instances (documents) used for evaluating the IR system effectiveness.

Each of the above accuracy metrics seeks to measure the quality of the proposed ranked model and the retrieved search results by this model. $\mathrm{P} @ \mathrm{~K}$ is used to measure the number of relevant documents in the top-k documents retrieved. However, this metric does not consider the graded relevance levels of each retrieved document, only if the query-document retrieved is relevant or not. MAP measures the average precision on the whole search results rather than the top-k query-document pairs retrieved. The NDCG@K metric considers the graded relevance level of each pair query-document for the top-k query-document retrieved. The difference between MAP and $R R @ K$ is that RR@K considers the impact of the position for each retrieved query-document pair in the search list more than MAP metric. Finally, MAE and RMSE calculate the difference between the relevance labels produced by the ranking model with the query-document pair features against the ground truth relevance labels. MAE and RMSE consider the ranking problem as a ranking and classification problem. In this paper, all these metrics are used in the performance comparison to aim for an extensive evaluation of the proposed LTR technique.

\subsection{Results and Discussion}

The variants of the proposed LTR method are called ES-Rank (baseline initilization), IESR-Rank (linear regression initialization) and IESVM-Rank (support vector machine initialization). Tables 4, 5, 6, 7 and 8 show the overall results for all the methods tested. The other fourteen methods are implemented in the packages RankLib (Dang, 2016), Sofiaml (Sculley, 2010), SVMRank (Joachims, 2016a), Layered Genetic Programming for LTR (RankGP) (Lin et al., 2007; Mick, 2016) and rt-rank for IGBRT (Mohan et al., 2011). There are no results for the GBRT technique in respect of MAP, P@10 and RR@10 due to 
the limitations of the rt-rank package for obtaining them. The parameter values used for those other approaches are the default settings in these packages. Those settings produced the shortest computational run time and the lowest memory size requirements for each approach. The experimental results presented are the average scores of five runs on 5 -folds cross validation. Each dataset fold consists of a training, a validation and a testing data. Experiments were conducted on a PC with $3.60 \mathrm{GHz}$ Intel (R) core(TM) i7-3820 CPU and $8 \mathrm{~GB}$ RAM. The implementation was in Java NetBeans under Windows 7 Enterprise Edition.

The results shown in tables 4, 5, 6, 7 and 8 correspond to the predictive values of the average performance of five runs by the tested approaches. As mentioned above, the performance is measured with the evaluation metrics MAP, NDCG@10,P@10,RR@10 and RMSE. From these results, it can be seen that IESRRank is generally the best approach producing the best performance among all methods in 7 out of 10 average MAP, 6 out of 10 average NDCG@10, 2 out of 10 average P@10, 2 out of 10 RR@10 and 4 out of 10 RMSE. The second best approach is ES-Rank, producing the best performance in 2 out of 10 average MAP, 2 out of 10 average NDCG@10, 1 out of 10 average P@10, 2 out of 10 average RR@10 and 2 out of 10 RMSE. Random Forest comes in the third position with 3 out of 10 average $\mathrm{P} @ 10$ and 3 out of 10 average RR@10, while IESVMRank is fourth with 4 out of 10 RMSE. The LambdaMART is fifth with 2 out of 10 average P@10 and 1 out of 10 average RR@10. The IGBRT and RankBoost are joint in the sixth position with 2 out of 10 average NDCG@10 for IGBRT, while RankBoost has 1 out of 10 average MAP and 1 out of 10 average P@10.

Figures 2, 3, 4, 5 and 6 illustrate the radar chart for each fitness evaluation metric results reported in the tables mentioned above. In the first four figures, higher values correspond to better performance, while in the last figure lower values correspond to better performance. From these figures it can be observed that the IESR-Rank technique exhibits the overall best performance among all techniques.

The statistical F-significant test of the results is presented in table 3 . This table shows the null hypothesis $\mathrm{P}$-values of the predictive results of the evaluation fitness metrics on the dataset folds. This F-test measures if there is differentiation between the average results between techniques or not, while its null hypothesis assumes that there is no difference between the results obtained by the techniques. If the p-values are small, this indicates that the hypothesis is rejected. The p-value under 0.05 indicates that the improvements for IESR-Rank and ES-Rank against the other LTR techniques are significant for distinguishing between them and the other techniques. From table 3 , the improvements in the results on MSLR-WEB10K, MQ2008, MQ2007, NP2003, HP2004 and TD2004 are significant, while the tests on Ohsumed, HP2003, TD2003 and NP2004 are not.

The average computational run-times of the algorithms for each benchmark dataset are shown in table 9 . These results show that the variants of the proposed LTR method are still very efficient in terms of computational runtime. It can be seen that by incorporating linear regression into ES-Rank, the computational run-time of IESR-Rank increases just slightly over ES-Rank, but as discussed above, the accuracy results produced by IESR-Rank are much better.

\section{Conclusion and Future Work}

This paper presented a new LTR approach that combines a $(1+1)$-Evolutionary Strategy with machine learning techniques. Three methods to initialize the first parent ranking function were tested. One method sets all weights to zero in the initial parent. The other two methods use Linear Regression and Support Vector Machine to create the 
Table 3: F-test of the predictive result for the algorithms on the datasets

\begin{tabular}{|c|c|c|c|c|c|}
\hline Dataset & MSLR-WEB10K & MQ2008 & MQ2007 & Ohsumed & HP2003 \\
\hline P-Value & 0.0001926 & 0.03833 & 0.04951 & 0.2649 & 0.0828 \\
\hline Dataset & TD2003 & NP2003 & HP2004 & TD2004 & NP2004 \\
\hline P-Value & 0.1945 & 0.02432 & 0.0159 & 0.01025 & 0.0564 \\
\hline
\end{tabular}

Table 4: Algorithms Average Performance Applied on 10 Datasets Using MAP Fitness Evaluation Metric

\begin{tabular}{|c|c|c|c|c|c|c|c|c|c|c|}
\hline Algorithm & MSLR-WEB10K & MQ2008 & MQ2007 & Ohsumed & HP2003 & TD2003 & NP2003 & HP2004 & TD2004 & NP2004 \\
\hline RankBoost & 0.5737 & 0.47722 & 0.45348 & 0.44784 & 0.69838 & 0.2053 & 0.64712 & 0.62586 & 0.2178 & 0.55286 \\
\hline SVMRank & 0.45736 & 0.39984 & 0.40784 & 0.38316 & 0.41926 & 0.0814 & 0.43316 & 0.3513 & 0.1241 & 0.37814 \\
\hline ListNET & 0.47346 & 0.45256 & 0.43964 & 0.4401 & 0.12414 & 0.0573 & 0.20114 & 0.17426 & 0.1357 & 0.15684 \\
\hline AdaRank & 0.57118 & 0.4653 & 0.45384 & 0.43656 & 0.72042 & 0.2452 & 0.61816 & 0.71532 & 0.1914 & 0.57006 \\
\hline MART & 0.57952 & 0.47324 & 0.45894 & 0.4269 & 0.74602 & 0.1877 & 0.66526 & 0.4995 & 0.2041 & 0.51884 \\
\hline Coordinate Ascent & 0.58628 & 0.48108 & 0.45976 & 0.44604 & 0.7477 & 0.2371 & 0.66282 & 0.6575 & 0.2245 & 0.65302 \\
\hline LambdaMART & 0.58574 & 0.4704 & 0.45522 & 0.4258 & 0.7373 & 0.1805 & 0.6545 & 0.50046 & 0.1874 & 0.49574 \\
\hline RankNET & 0.48584 & 0.45198 & 0.44808 & 0.4351 & 0.73694 & 0.2242 & 0.64972 & 0.61946 & 0.1863 & 0.64738 \\
\hline Random Forest & 0.59818 & 0.4699 & 0.45866 & 0.43252 & 0.76856 & 0.2847 & 0.70794 & 0.62994 & 0.2541 & 0.60302 \\
\hline Linear Regression & 0.502 & 0.455 & 0.42974 & 0.4333 & 0.49246 & 0.217 & 0.55652 & 0.5063 & 0.1886 & 0.46708 \\
\hline RankGP & 0.46732 & 0.42672 & 0.41424 & 0.39914 & 0.56378 & 0.2149 & 0.58136 & 0.52558 & 0.2104 & 0.5142 \\
\hline CoRR & 0.47614 & 0.43946 & 0.42164 & 0.39604 & 0.48888 & 0.2244 & 0.56786 & 0.54346 & 0.2103 & 0.4865 \\
\hline LambdaRank & 0.47574 & 0.34836 & 0.3398 & 0.30728 & 0.71664 & 0.1309 & 0.64516 & 0.36672 & 0.1721 & 0.64424 \\
\hline ES-Rank & 0.570386 & 0.48324 & 0.47004 & 0.42162 & 0.79902 & 0.2784 & 0.74894 & 0.71794 & 0.2615 & 0.75228 \\
\hline IESR-Rank & 0.60272 & 0.49366 & 0.47312 & 0.4348 & 0.8002 & 0.2914 & 0.75444 & 0.69264 & 0.2584 & 0.75792 \\
\hline IESVM-Rank & 0.45742 & 0.47336 & 0.4555 & 0.4432 & 0.63686 & 0.2535 & 0.66258 & 0.57474 & 0.1932 & 0.51956 \\
\hline
\end{tabular}

Table 5: Algorithms Average Performance Applied on 10 Datasets Using NDCG@10 Fitness Evaluation Metric

\begin{tabular}{|c|c|c|c|c|c|c|c|c|c|c|}
\hline Algorithm & MSLR-WEB10K & MQ2008 & MQ2007 & Ohsumed & HP2003 & TD2003 & NP2003 & HP2004 & TD2004 & NP2004 \\
\hline RankBoost & 0.3353 & 0.50032 & 0.4328 & 0.43852 & 0.74472 & 0.27468 & 0.68082 & 0.6772 & 0.30872 & 0.63046 \\
\hline SVMRank & 0.22156 & 0.43222 & 0.36458 & 0.33296 & 0.44236 & 0.10658 & 0.47888 & 0.3468 & 0.19726 & 0.43358 \\
\hline ListNET & 0.19274 & 0.484 & 0.4169 & 0.39254 & 0.16592 & 0.1213 & 0.17876 & 0.18366 & 0.11386 & 0.27818 \\
\hline AdaRank & 0.34624 & 0.49662 & 0.42998 & 0.4478 & 0.74636 & 0.26516 & 0.65446 & 0.7132 & 0.27936 & 0.62576 \\
\hline MART & 0.3947 & 0.50362 & 0.4398 & 0.42822 & 0.78748 & 0.27564 & 0.70846 & 0.54834 & 0.24478 & 0.5874 \\
\hline Coordinate Ascent & 0.40156 & 0.50668 & 0.44262 & 0.4522 & 0.77722 & 0.31892 & 0.74042 & 0.77236 & 0.31526 & 0.70222 \\
\hline LambdaMART & 0.39996 & 0.5053 & 0.44776 & 0.41652 & 0.7775 & 0.2841 & 0.6955 & 0.62498 & 0.25028 & 0.50522 \\
\hline RankNET & 0.19148 & 0.4839 & 0.42428 & 0.44138 & 0.76688 & 0.22008 & 0.6858 & 0.6632 & 0.25882 & 0.7223 \\
\hline Random Forest & 0.39964 & 0.49676 & 0.43938 & 0.4377 & 0.79764 & 0.362 & 0.7548 & 0.6645 & 0.34918 & 0.63992 \\
\hline Linear Regression & 0.36118 & 0.48712 & 0.41972 & 0.43024 & 0.55242 & 0.32034 & 0.61064 & 0.55558 & 0.27498 & 0.54092 \\
\hline RankGP & 0.35368 & 0.44062 & 0.41546 & 0.41372 & 0.59298 & 0.2543 & 0.5859 & 0.66958 & 0.2726 & 0.71198 \\
\hline CoRR & 0.35758 & 0.47406 & 0.42248 & 0.4243 & 0.56956 & 0.25054 & 0.55668 & 0.6589 & 0.2786 & 0.59868 \\
\hline LambdaRank & 0.19592 & 0.3125 & 0.27558 & 0.28014 & 0.72216 & 0.15086 & 0.68602 & 0.2947 & 0.13016 & 0.53932 \\
\hline IGBRT & 0.39424 & 0.51814 & 0.45722 & 0.4437 & 0.80816 & 0.3062 & 0.756326 & NA & NA & NA \\
\hline ES-Rank & 0.38234 & 0.50656 & 0.45062 & 0.4461 & 0.82616 & 0.36244 & 0.753792 & 0.77784 & 0.35792 & 0.79224 \\
\hline IESR-Rank & 0.41504 & 0.5169 & 0.45506 & 0.4544 & 0.82866 & 0.3761 & 0.75752 & 0.77806 & 0.3557 & 0.7899 \\
\hline IESVM-Rank & 0.22404 & 0.49762 & 0.43558 & 0.44854 & 0.7885 & 0.3402 & 0.73256 & 0.57188 & 0.21326 & 0.52362 \\
\hline
\end{tabular}




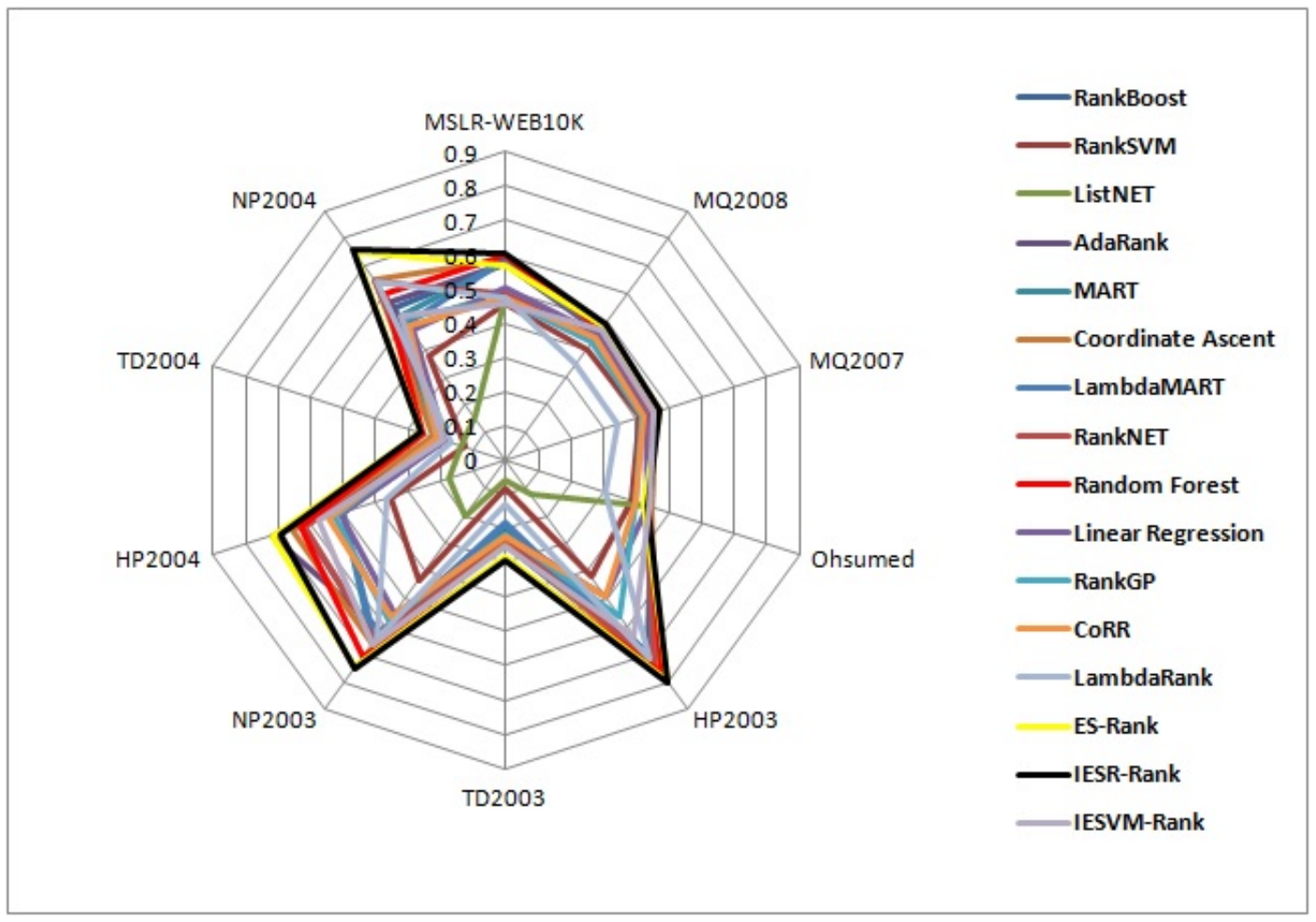

Fig. 2: Illustrating the MAP performance for all LTR methods on the LETOR datasets.

Table 6: Algorithms Average Performance Applied on 10 Datasets Using P@10 Fitness Evaluation Metric

\begin{tabular}{|c|c|c|c|c|c|c|c|c|c|c|}
\hline Algorithm & MSLR-WEB10K & MQ2008 & MQ2007 & Ohsumed & HP2003 & TD2003 & NP2003 & HP2004 & TD2004 & NP2004 \\
\hline RankBoost & 0.58678 & 0.27376 & 0.37164 & 0.50412 & 0.10202 & 0.144 & 0.088 & 0.08334 & 0.23334 & 0.08534 \\
\hline SVMRank & 0.40454 & 0.2501 & 0.33194 & 0.40128 & 0.06454 & 0.072 & 0.06758 & 0.05334 & 0.14816 & 0.05334 \\
\hline ListNET & 0.43586 & 0.267 & 0.3582 & 0.46556 & 0.03334 & 0.068 & 0.03298 & 0.0242 & 0.112 & 0.02666 \\
\hline AdaRank & 0.59416 & 0.24738 & 0.35558 & 0.49852 & 0.1 & 0.128 & 0.08532 & 0.08268 & 0.224 & 0.08934 \\
\hline MART & 0.63118 & 0.27502 & 0.37872 & 0.47554 & 0.104 & 0.146 & 0.084 & 0.08198 & 0.23734 & 0.08136 \\
\hline Coordinate Ascent & 0.62682 & 0.27328 & 0.37768 & 0.4831 & 0.10334 & 0.158 & 0.09162 & 0.09732 & 0.24932 & 0.092 \\
\hline LambdaMART & 0.64484 & 0.27504 & 0.38378 & 0.47804 & 0.09598 & 0.156 & 0.08696 & 0.07866 & 0.22932 & 0.07468 \\
\hline RankNET & 0.44282 & 0.26686 & 0.36182 & 0.49566 & 0.09734 & 0.148 & 0.08732 & 0.08464 & 0.21468 & 0.096 \\
\hline Random Forest & 0.60724 & 0.27466 & 0.37824 & 0.49174 & 0.10536 & 0.194 & 0.09362 & 0.08666 & 0.26668 & 0.08798 \\
\hline Linear Regression & 0.45732 & 0.2735 & 0.3724 & 0.48078 & 0.08666 & 0.18 & 0.08296 & 0.08002 & 0.22532 & 0.08134 \\
\hline RankGP & 0.44742 & 0.24002 & 0.34394 & 0.4155 & 0.06734 & 0.10532 & 0.082 & 0.07332 & 0.1426 & 0.07334 \\
\hline CoRR & 0.44112 & 0.24276 & 0.35456 & 0.41784 & 0.07734 & 0.08 & 0.068 & 0.05866 & 0.11114 & 0.05602 \\
\hline LambdaRank & 0.43016 & 0.21268 & 0.29236 & 0.32832 & 0.05602 & 0.022 & 0.03248 & 0.02132 & 0.13202 & 0.04346 \\
\hline ES-Rank & 0.63446 & 0.27018 & 0.37684 & 0.49358 & 0.09746 & 0.184 & 0.09564 & 0.09598 & 0.25732 & 0.09066 \\
\hline IESR-Rank & 0.64338 & 0.2753 & 0.37208 & 0.4976 & 0.10406 & 0.19 & 0.08902 & 0.09874 & 0.25884 & 0.09066 \\
\hline IESVM-Rank & 0.40536 & 0.27182 & 0.37576 & 0.4814 & 0.10266 & 0.166 & 0.09156 & 0.08266 & 0.168 & 0.06868 \\
\hline
\end{tabular}




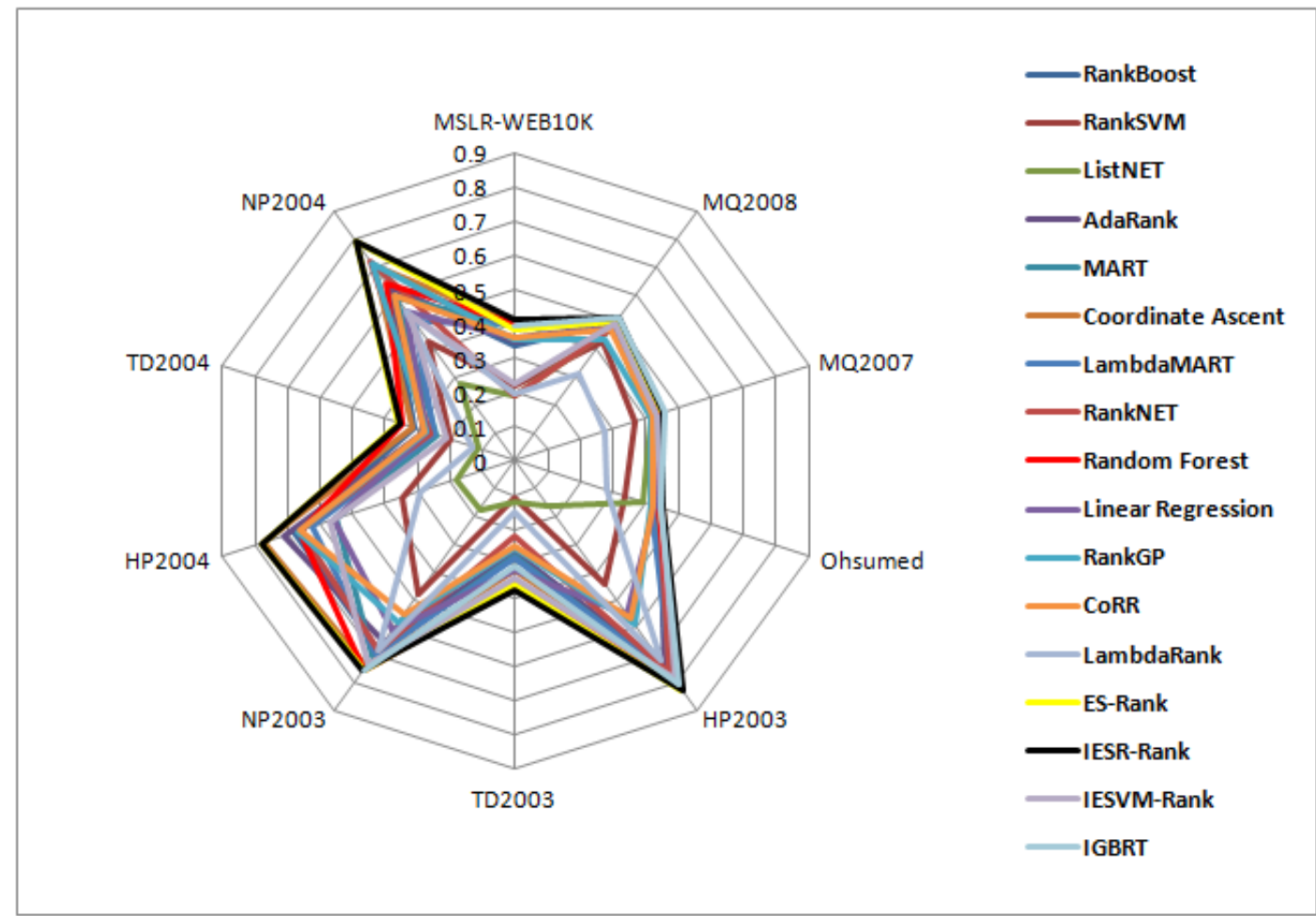

Fig. 3: Illustrating the NDCG@10 performance for all LTR methods on the LETOR datasets.

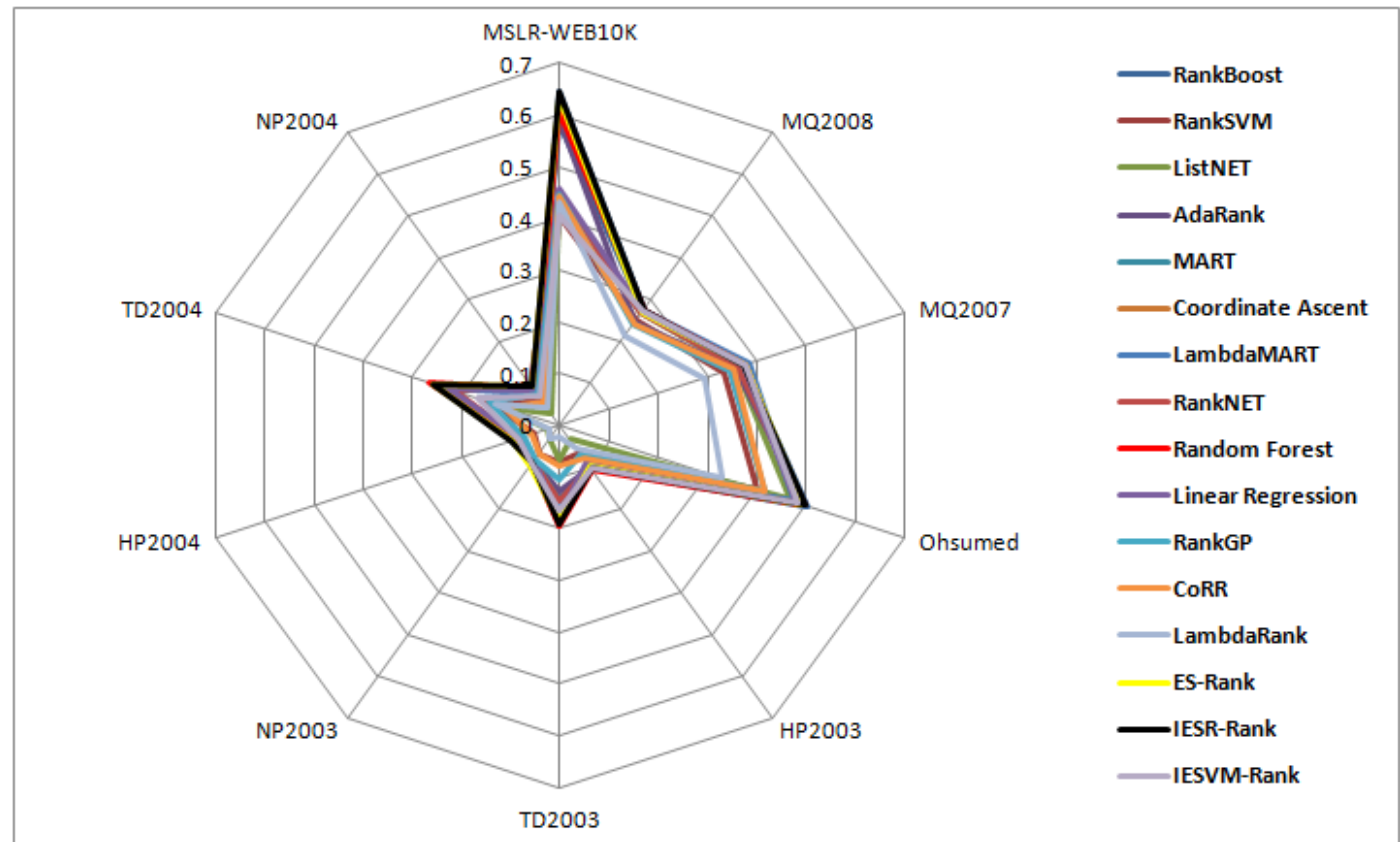

Fig. 4: Illustrating the P@10 performance for all LTR methods on the LETOR datasets. 


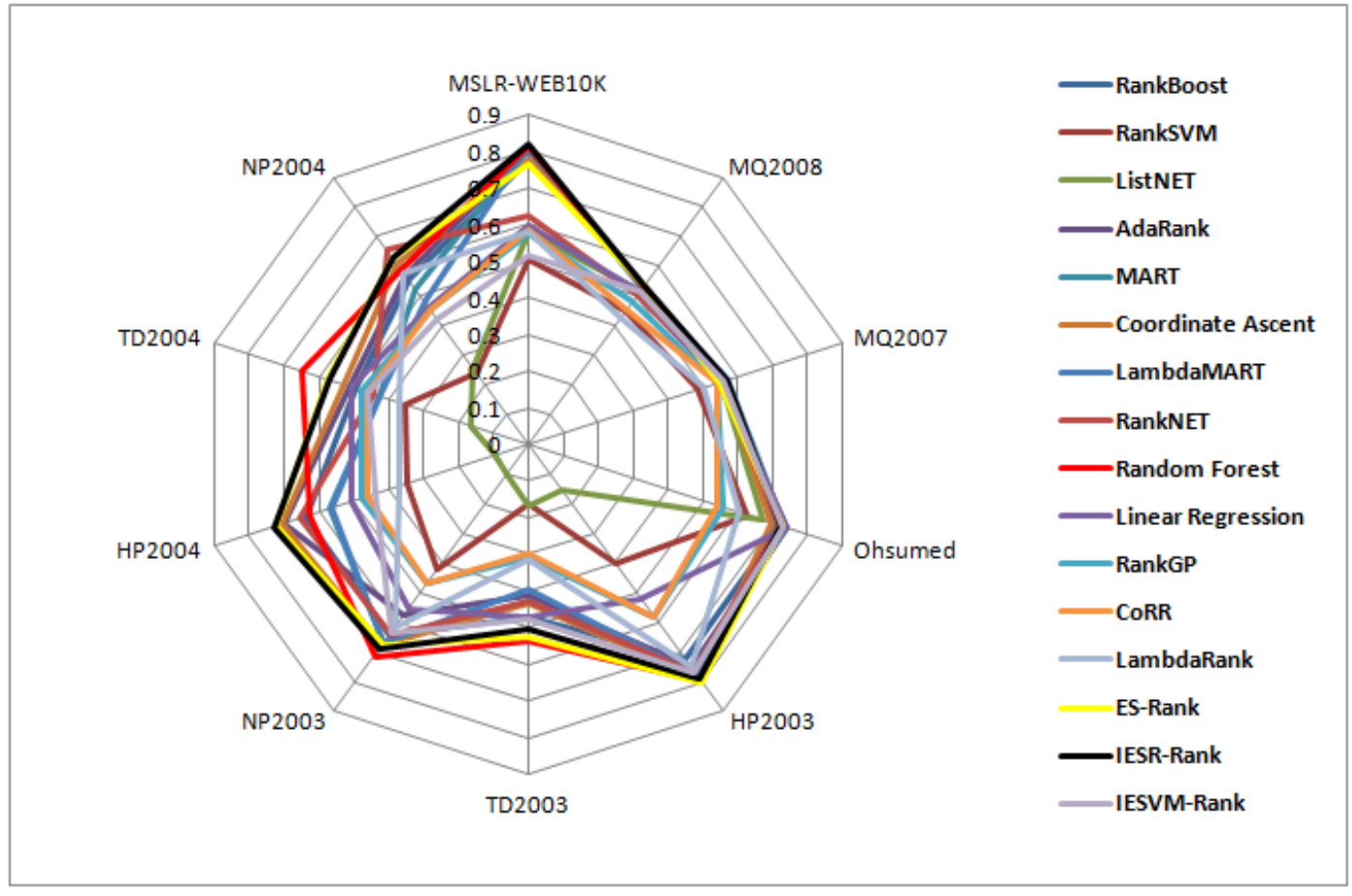

Fig. 5: Illustrating the RR@10 performance for all LTR methods on the LETOR datasets.

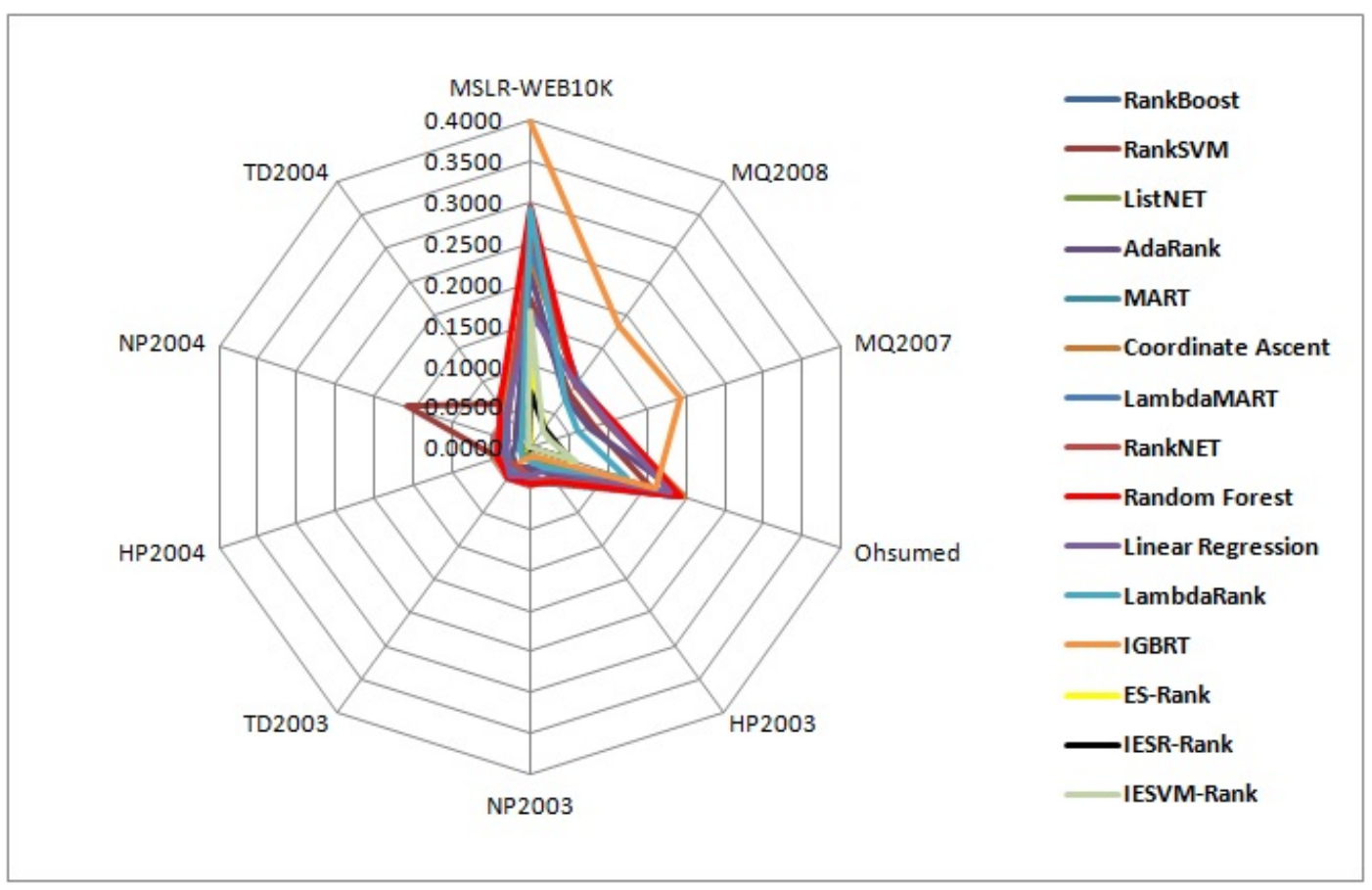

Fig. 6: Illustrating the RMSE performance for all LTR methods on the LETOR datasets. 
Table 7: Algorithms Average Performance Applied on 10 Datasets Using RR@10 Fitness Evaluation Metric

\begin{tabular}{|c|c|c|c|c|c|c|c|c|c|c|}
\hline Algorithm & MSLR-WEB10K & MQ2008 & MQ2007 & Ohsumed & HP2003 & TD2003 & NP2003 & HP2004 & TD2004 & NP2004 \\
\hline RankBoost & 0.77662 & 0.5331 & 0.5638 & 0.72272 & 0.72594 & 0.46966 & 0.64464 & 0.63994 & 0.49766 & 0.55688 \\
\hline SVMRank & 0.50512 & 0.45052 & 0.48534 & 0.62528 & 0.40398 & 0.16098 & 0.42384 & 0.34858 & 0.35466 & 0.24216 \\
\hline ListNET & 0.58142 & 0.51342 & 0.55174 & 0.66964 & 0.1545 & 0.16908 & 0.10356 & 0.09628 & 0.1631 & 0.2565 \\
\hline AdaRank & 0.8025 & 0.53318 & 0.54832 & 0.73908 & 0.75232 & 0.41014 & 0.57586 & 0.6979 & 0.50998 & 0.5683 \\
\hline MART & 0.80944 & 0.5295 & 0.5691 & 0.70606 & 0.79212 & 0.4297 & 0.66808 & 0.56728 & 0.42806 & 0.52258 \\
\hline Coordinate Ascent & 0.77194 & 0.53348 & 0.55776 & 0.69728 & 0.7889 & 0.43212 & 0.67758 & 0.70362 & 0.52898 & 0.60846 \\
\hline LambdaMART & 0.81202 & 0.52962 & 0.57028 & 0.73662 & 0.77454 & 0.39706 & 0.67244 & 0.56102 & 0.42394 & 0.48148 \\
\hline RankNET & 0.62222 & 0.50464 & 0.55222 & 0.71924 & 0.76682 & 0.42584 & 0.63974 & 0.65254 & 0.4467 & 0.6554 \\
\hline Random Forest & 0.81182 & 0.53046 & 0.5661 & 0.72284 & 0.7961 & 0.53858 & 0.7134 & 0.62522 & 0.65104 & 0.58924 \\
\hline Linear Regression & 0.59422 & 0.51336 & 0.55044 & 0.74138 & 0.51912 & 0.47096 & 0.55356 & 0.50648 & 0.50404 & 0.46376 \\
\hline RankGP & 0.57304 & 0.4832 & 0.5418 & 0.55918 & 0.58422 & 0.30924 & 0.46534 & 0.47744 & 0.47764 & 0.45378 \\
\hline CoRR & 0.58562 & 0.45536 & 0.54334 & 0.54078 & 0.58076 & 0.29544 & 0.46624 & 0.4637 & 0.46058 & 0.4519 \\
\hline LambdaRank & 0.58 & 0.42796 & 0.5001 & 0.602 & 0.75228 & 0.31154 & 0.61492 & 0.36758 & 0.36956 & 0.57872 \\
\hline ES-Rank & 0.764114 & 0.53714 & 0.54626 & 0.72654 & 0.80106 & 0.5239 & 0.67388 & 0.72378 & 0.57316 & 0.6288 \\
\hline IESR-Rank & 0.81912 & 0.5352 & 0.56856 & 0.72458 & 0.79182 & 0.50572 & 0.69022 & 0.72912 & 0.5701 & 0.6293 \\
\hline IESVM-Rank & 0.5127 & 0.51288 & 0.55996 & 0.72912 & 0.7679 & 0.47424 & 0.6383 & 0.43534 & 0.45852 & 0.42166 \\
\hline
\end{tabular}

Table 8: Algorithms Performance Applied on 10 Datasets Using RMSE Fitness Evaluation Metric

\begin{tabular}{|c|c|c|c|c|c|c|c|c|c|c|}
\hline Algorithms & MSLR-WEB10K & MQ2008 & MQ2007 & Ohsumed & HP2003 & NP2003 & TD2003 & HP2004 & NP2004 & TD2004 \\
\hline RankBoost & 0.2319 & 0.0973 & 0.1018 & 0.1905 & 0.0511 & 0.0409 & 0.0397 & 0.0409 & 0.0362 & 0.0496 \\
\hline SVMRank & 0.1820 & 0.0795 & 0.0820 & 0.1536 & 0.0324 & 0.0282 & 0.0268 & 0.0434 & 0.1597 & 0.0667 \\
\hline ListNET & 0.2735 & 0.0934 & 0.0985 & 0.1912 & 0.0183 & 0.0113 & 0.0128 & 0.0048 & 0.0124 & 0.0245 \\
\hline AdaRank & 0.2127 & 0.0742 & 0.0755 & 0.1985 & 0.0402 & 0.0245 & 0.0301 & 0.0227 & 0.0184 & 0.0288 \\
\hline MART & 0.2300 & 0.0980 & 0.1045 & 0.1959 & 0.0567 & 0.0420 & 0.0367 & 0.0297 & 0.0340 & 0.0459 \\
\hline Coordinate Ascent & 0.2275 & 0.0975 & 0.1017 & 0.1987 & 0.0521 & 0.0423 & 0.0408 & 0.0457 & 0.0429 & 0.0554 \\
\hline LambdaMART & 0.2400 & 0.0984 & 0.1044 & 0.1916 & 0.0512 & 0.0432 & 0.0344 & 0.0373 & 0.0300 & 0.0458 \\
\hline RankNET & 0.2647 & 0.0931 & 0.0984 & 0.1884 & 0.0491 & 0.0404 & 0.0272 & 0.0418 & 0.0436 & 0.0451 \\
\hline Random Forest & 0.2948 & 0.0964 & 0.1046 & 0.1937 & 0.0533 & 0.0446 & 0.0477 & 0.0409 & 0.0388 & 0.0633 \\
\hline Linear Regression & 0.1653 & 0.0952 & 0.0994 & 0.1795 & 0.0340 & 0.0352 & 0.0428 & 0.0325 & 0.0297 & 0.0491 \\
\hline LambdaRank & 0.2910 & 0.0718 & 0.0620 & 0.1237 & 0.0312 & 0.0167 & 0.0170 & 0.0119 & 0.0122 & 0.0188 \\
\hline IGBRT & 0.3968 & 0.1833 & 0.1931 & 0.1622 & 0.0158 & 0.0138 & 0.0205 & NA & NA & NA \\
\hline ES-Rank & 0.0953 & 0.0295 & 0.0282 & 0.0561 & 0.0012 & 0.0017 & 0.0028 & 0.0009 & 0.0000 & 0.0014 \\
\hline IESR-Rank & 0.0681 & 0.0284 & 0.0292 & 0.0504 & 0.0003 & 0.0002 & 0.0019 & 0.0003 & 0.0026 & 0.0022 \\
\hline IESVM-Rank & 0.1655 & 0.0260 & 0.0266 & 0.0580 & 0.0005 & 0.0001 & 0.0006 & 0.0010 & 0.0032 & 0.0050 \\
\hline
\end{tabular}

initial parent. Then, the parent is evolved for a number of generations by the evolutionary strategy. The performance of the proposed approach was compared to fourteen other machine learning and computational intelligence approaches from the literature. The metrics Mean Average Precision (MAP), Normalized Discounted Cumulative Gain (NDCG@10), Precision (P@10), Reciprocal Ranking(RR@10) and Root Mean Square Error (RMSE) were used as fitness functions within the proposed method and also for evaluating the performance of the LTR approaches in the comparison. The benchmark datasets used here are: MSLR-WEB10K (Microsoft Bing ten thousand web queries) dataset, LETOR 4 (MQ2008, MQ2007 TREC Million queries datasets for years 2008 and 2007) and LETOR 3 (Ohsumed and 6 .Gov datasets).

From the experimental results, the overall conclusion is that the variant of the proposed method that uses Linear Regression exhibited better performance than the other methods tested. This variant called IESR-Rank achieved the best performance in 7 out of 10 average MAP, 6 out of 10 average NDCG@10, 2 out of 10 average $\mathrm{P} @ 10,2$ out of 10 average RR@10 and 4 out of 10 RMSE. The second best overall performance was exhibited by the variant ES-Rank which simply sets all 
Table 9: Average run-times of the five evaluation fitness metrics measured in seconds for the algorithms

\begin{tabular}{|c|l|c|c|c|c|c|c|c|c|c|}
\hline Algorithm & $\begin{array}{l}\text { MSLR- } \\
\text { WEB10K }\end{array}$ & MQ2008 & MQ2007 & Ohsumed & HP2003 & NP2003 & TD2003 & HP2004 & NP2004 & TD2004 \\
\hline RankBoost & 3720 & 15 & 74 & 28 & 483 & 1153 & 460 & 493 & 597 & 604 \\
\hline SVMRank & 32409 & 19 & 23 & 15 & 33 & 40 & 36 & 33 & 35 & 32 \\
\hline ListNET & 18005 & 45 & 95 & 43 & 145 & 255 & 250 & 145 & 140 & 142 \\
\hline AdaRank & 3600 & 11 & 20 & 16 & 228 & 453 & 486 & 227 & 123 & 240 \\
\hline MART & 1200 & 8 & 11 & 12 & 12 & 23 & 11 & 13 & 15 & 19 \\
\hline CA & 25200 & 37 & 240 & 28 & 580 & 940 & 396 & 460 & 480 & 460 \\
\hline LambdaMART & 3720 & 9 & 11 & 8 & 24 & 89 & 21 & 23 & 25 & 27 \\
\hline RankNET & 10800 & 33 & 96 & 98 & 55 & 119 & 130 & 110 & 117 & 298 \\
\hline RF & 3660 & 27 & 55 & 17 & 72 & 168 & 71 & 72 & 70 & 80 \\
\hline LR & 157 & 2 & 3 & 3 & 5 & 6 & 5 & 5 & 4 & 5 \\
\hline RankGP & 26020 & 375 & 390 & 360 & 430 & 519 & 486 & 423 & 406 & 496 \\
\hline CoRR & 10803 & 42 & 51 & 39 & 59 & 61 & 58 & 57 & 58 & 57 \\
\hline LambdaRank & 18015 & 46 & 142 & 165 & 145 & 237 & 462 & 150 & 150 & 438 \\
\hline IGBRT & 36750 & 274 & 253 & 197 & 393 & 389 & 386 & NA & NA & NA \\
\hline ES-Rank & 1800 & 35 & 51 & 15 & 128 & 137 & 47 & 69 & 68 & 70 \\
\hline IESR-Rank & 1957 & 37 & 54 & 18 & 133 & 143 & 52 & 74 & 72 & 75 \\
\hline IESVM-Rank & 34209 & 54 & 74 & 30 & 161 & 177 & 83 & 102 & 103 & 102 \\
\hline
\end{tabular}

initial weights to zero in the initial parent. Random Forest comes in the third position, IESVM-Rank showed the fourth best performance followed by LambdaMART. IGBRT and RankBoost are joint in the sixth position followed by RankBoost. Thus, the proposed method combining evolutionary computation with machine learning is a competitive approach to tackle the LTR problem in information retrieval. It is clear from the results in this paper that a hybrid LTR technique that combines optimisation (an evolutionary strategy) with machine learning (linear regression and support vector machines) strikes a good balance between effectiveness and computational efficiency. Despite the proposed IESR-Rank, ES-Rank and IESVM-Rank performing better overall against the default settings of the other fourteen techniques, it is unclear whether such performance will hold after sophisticated tuning of the other techniques. Future research should seek to develop enhanced versions of the proposed approach by investigating other optimisation methods besides evolutionary strategies. For example, other heuristic optimisation paradigms like simulated annealing, late acceptance hill-climbing, great deluge and others could be used in combination with linear regression. It is argued that the combination of optimisation and machine learning is a fertile ground for the development of high-performance LTR methods for information retrieval.

\section{Compliance with ethical standards}

Conflict of interest The authors declare that they have no conflict of interest.

\section{References}

Ricardo A. Baeza-Yates and Berthier A. Ribeiro-Neto. Modern Information Retrieval - the concepts and technology behind search. Pearson Education Ltd., Harlow, England, 2nd edition edition, 2011.

Hans-George Beyer and Hans-Paul Schwefel. Evolution strategies - A comprehensive introduction. Natural Computing, 1:3-52, 2002.

Leo Breiman. Random forests. Machine Learning, 45(1):5-32, 2001. ISSN 1573-0565. 
doi: 10.1023/A:1010933404324. URL http: //dx.doi.org/10.1023/A:1010933404324.

Jason Brownlee. Overfitting and underfitting with machine learning algorithms, 2017. URL https: //machinelearningmastery.com/ overfitting-and-underfitting-withmachine-learning-algorithms/.

Chris Burges, Tal Shaked, Erin Renshaw, Ari Lazier, Matt Deeds, Nicole Hamilton, and Greg Hullender. Learning to rank using gradient descent. In Proceedings of the 22Nd International Conference on Machine Learning, ICML '05, pages 89-96, New York, NY, USA, 2005. ACM. ISBN 1-59593-180-5. doi: 10.1145/1102351.1102363. URL http: //doi.acm.org/10.1145/1102351.1102363.

Christopher J. C. Burges. From RankNet to LambdaRank to LambdaMART: An overview. Technical report, Microsoft Research, 2010. URL http://research.microsoft.com/en-us/ um/people/cburges/tech_reports/MSRTR-2010-82.pdf.

Zhe Cao, Tao Qin, Tie-Yan Liu, Ming-Feng Tsai, and Hang Li. Learning to rank: from pairwise approach to listwise approach. In Proceedings of the 24th international conference on Machine learning, ICML '07, pages 129-136, New York, NY, USA, 2007. ACM. ISBN 978-1-59593-793-3. doi: 10.1145/1273496.1273513. URL http:// doi.acm.org/10.1145/1273496.1273513.

Olivier Chapelle and Yi Chang. Yahoo! learning to rank challenge overview. In Proceedings of the Yahoo! Learning to Rank Challenge, held at ICML 2010, Haifa, Israel, June 25, 2010, pages 1-24, 2011. URL http://www.jmlr.org/proceedings/ papers/v14/chapelle11a.html.

Van Dang. RankLib, http://www.cs.umass.edu/ṽdang/ranklib.html, 2016. URL http://www.cs.umass.edu/ $\sim\{\}$ vdang/ranklib.html.

Pedro A Diaz-Gomez and Dean F Hougen. Initial population for genetic algorithms: A metric approach. In Proceedings of the 2007
International Conference on Genetic and Evolutionary Methods GEM, pages 43-49, 2007.

Yoav Freund, Raj Iyer, Robert E. Schapire, and Yoram Singer. An efficient boosting algorithm for combining preferences. Journal of Machine Learning Research, 4:933-969, December 2003. ISSN 1532-4435. URL http://dl.acm.org/citation.cfm?id= 945365.964285.

Jerome H. Friedman. Greedy function approximation: A gradient boosting machine. The Annals of Statistics, 29(5):1189-1232, 2001. ISSN 00905364. URL http:// www.jstor.org/stable/2699986.

O. Ali Sadek Ibrahim and D. Landa-Silva. Term frequency with average term occurrences for textual information retrieval. Soft Computing, 20(8):3045-3061, 2016. ISSN 1433-7479. doi: 10.1007/s00500-015-1935-7. URL http://dx.doi.org/10.1007/s00500015-1935-7.

Osman Ali Sadek Ibrahim and Dario LandaSilva. Es-rank: Evolution strategy learning to rank approach. In Proceedings of the Symposium on Applied Computing, SAC '17, pages 944-950, New York, NY, USA, 2017. ACM. ISBN 978-1-4503-4486-9. doi: 10.1145/3019612.3019696. URL http:// doi.acm.org/10.1145/3019612.3019696.

Mohammad Ashiful Islam. Rankgpes: Learning to rank for information retrieval using a hybrid genetic programming with evolutionary strategies, 2013.

Thorsten Joachims. Support vector machine for ranking, 2016a. URL https : //www.cs.cornell.edu/people/tj/ svm_light/svm_rank.html\#References.

Thorsten Joachims. Svmlight: Support vector machine for classification and ranking, 2016b. URL http://svmlight.joachims.org/.

Hang Li. Learning to Rank for Information Retrieval and Natural Language Processing, Second Edition. Morgan \& Claypool Publishers, 2014. ISBN 9781627055857. 
J. Y. Lin, J. Y. Yeh, and Chao Chung Liu. Learning to rank for information retrieval using layered multi-population genetic programming. In Computational Intelligence and Cybernetics (CyberneticsCom), 2012 IEEE International Conference on, pages 45-49, July 2012. doi: 10.1109/ CyberneticsCom.2012.6381614.

Jung-Yi Lin, Hao-Ren Ke, Been-Chian Chien, and Wei-Pang Yang. Designing a classifier by a layered multi-population genetic programming approach. Pattern Recognition, 40(8):2211-2225, 2007.

Tie-Yan Liu. Learning to rank for information retrieval. Foundation Trends of Information Retrieval, 3(3):225-331, March 2009. ISSN 1554-0669. doi: 10.1561/1500000016.

Tie-Yan Liu. Learning to Rank for Information Retrieval, chapter The LETOR Datasets, pages 133-143. Springer Berlin Heidelberg, Berlin, Heidelberg, 2011. ISBN 978-3-642-14267-3. doi: 10.1007/978-3642-14267-3_10. URL http://dx.doi.org/ 10.1007/978-3-642-14267-3_10.

Christopher D. Manning, Prabhakar Raghavan, and Hinrich Schütze. Introduction to Information Retrieval. Cambridge University Press, New York, NY, USA, 2008. ISBN 0521865719, 9780521865715 .

Donald Metzler and W. Bruce Croft. Linear feature-based models for information retrieval. Information Retrieval, 10(3): 257-274, 2007. ISSN 1573-7659. doi: 10.1007/s10791-006-9019-z. URL http:// dx.doi.org/10.1007/s10791-006-9019-z.

Jung-Yi Lin Mick, 2016. URL http: //people.cs.nctu.edu.tw/ jylin/lagep/ lagep.html.

Steven J. Miller. The method of least squares, 2006. URL http: //citeseerx.ist.psu.edu/viewdoc/ summary?doi=10.1.1.710.4069.

A. Mohan, Z. Chen, and K.Q. Weinberger. Web-search ranking with initialized gradient boosted regression trees. In Journal of Machine Learning Research, Workshop and Conference Proceedings, volume 14, pages
77-89, 2011.

Tao Qin and Tie-Yan Liu. Introducing LETOR 4.0 datasets. CoRR, abs/1306.2597, 2013. URL http: //arxiv.org/abs/1306.2597.

Tao Qin, Tie-Yan Liu, Jun Xu, and Hang Li. Letor: A benchmark collection for research on learning to rank for information retrieval. Information Retrieval, 13(4):346-374, 2010. ISSN 1573-7659. doi: 10.1007/s10791-0099123-y. URL http://dx.doi.org/10.1007/ s10791-009-9123-y.

D. Sculley. Combined regression and ranking. In Proceedings of the 16th ACM SIGKDD International Conference on Knowledge Discovery and Data Mining, KDD '10, pages 979-988, New York, NY, USA, 2010. ACM. ISBN 978-1-4503-0055-1. doi: 10.1145/1835804.1835928. URL http:// doi.acm.org/10.1145/1835804.1835928.

Alberto Tonon, Gianluca Demartini, and Philippe Cudr-Mauroux. Pooling-based continuous evaluation of information retrieval systems. Information Retrieval Journal, 18 (5):445-472, 2015. ISSN 1386-4564. doi: 10.1007/s10791-015-9266-y.

Julián Urbano. Test collection reliability: a study of bias and robustness to statistical assumptions via stochastic simulation. Information Retrieval Journal, 19 (3):313-350, 2016. ISSN 1573-7659. doi: 10.1007/s10791-015-9274-y. URL http:// dx.doi.org/10.1007/s10791-015-9274-y.

Jun $\mathrm{Xu}$ and Hang Li. Adarank: A boosting algorithm for information retrieval. In Proceedings of the 30th Annual International ACM SIGIR Conference on Research and Development in Information Retrieval, SIGIR '07, pages 391-398, New York, NY, USA, 2007. ACM. ISBN 978-1-59593-597-7. doi: 10.1145/1277741.1277809. URL http: //doi.acm.org/10.1145/1277741.1277809.

Xin Yan and Xiao Gang Su. Linear Regression Analysis: Theory and Computing. World Scientific Publishing Co., Inc., River Edge, NJ, USA, 2009. ISBN 9789812834102, 9812834109. 